\title{
INSTALLATION AND COMMISSIONING OF THE NEW 150 KW PLANT FOR THE ELETTRA RF SYSTEM UPGRADE
}

\author{
A. Fabris, M. Bocciai, C. Pasotti, M. Rinaldi, \\ Sincrotrone Trieste, S.S.14 - km.163,5, in AREA Science Park, 34012 Basovizza, Trieste, Italy
}

\section{Abstract}

Elettra is the Italian third generation light source in operation in Trieste since 1993. The project of upgrade of the Elettra RF system has become necessary to provide the needed operating margins when all the insertion devices are operational and in view of possible increases in beam current and energy. The first phase of the project regards one of the four plants, which has been upgraded from 60 to $150 \mathrm{~kW} \mathrm{cw}$. The power amplifier has been built combining two $80 \mathrm{~kW}$ IOTs (inductive output tubes) by means of a switchless combiner. The amplifier and the power plant components have been installed in the second half of year 2006. A coaxial to waveguide transition has been specially designed to interface the coaxial coupler of the cavity to the waveguide power transmission system, taking into account the risks connected to power from the higher order modes excited by the beam in the cavity. After giving an overview of the project, this paper discusses the technical choices adopted, the tests performed during the installation phase and the commissioning of the new system with beam during machine operation.

\section{INTRODUCTION}

The original RF system of Elettra (installed in 1993) was composed of four $60 \mathrm{~kW} 500 \mathrm{MHz}$ plants, with a TV klystron as the final amplifying stage. Each plant powered a single cell copper cavity.

During the years, the increase in the number of insertion devices has considerably raised the power requirements. Now they amount to $121 \mathrm{~kW}$ at $2 \mathrm{GeV}$ if one takes into account the superconducting wiggler. The increase in beam power was generally compensated by decreasing the cavity gap voltage with a consequent decrease in beam lifetime. In addition, this increase also has spoiled the safety margins in the RF system operation.

For all these reasons a project of upgrade of the RF system was started to provide more power to the beam (also in view of further upgrades in beam current or energy), a higher lifetime and to restore and widen the operating margins of the RF system [1].

\section{TECHNICAL CHOICES}

The design strategy of the project has taken into account the following requirements:

- Minimum interference with machine operation. This leads to a gradual approach and the requirement of keeping unchanged the space for RF components in the machine. This also means that it is not possible to increase the number of the cavities or alternatively to install superconducting cavities, which would require the removal of one insertion device.

- Consistency with other upgrades of the facility and particularly the new injector under construction [2], where a $30 \mathrm{~kW}$ RF plant is required.

All this has lead to a three stages upgrade program:

- $\quad$ Phase A: upgrade to $150 \mathrm{~kW}$ of one plant. The old $60 \mathrm{~kW}$ will be used for the new booster RF plant.

- $\quad$ Phase B: upgrade to $150 \mathrm{~kW}$ of a second plant. The $60 \mathrm{~kW}$ plant will be moved to the booster for redundancy.

- $\quad$ Phase C: upgrade the remaining two plants to 150 $\mathrm{kW}$. At the end of phase $\mathrm{C}$, the available $\mathrm{RF}$ power will be $600 \mathrm{~kW}$, i.e. 2.5 times the original value.

At the time being phase A has been implemented.

A detailed analysis of the possibilities for the power amplifier was performed, taking also into consideration the time scales of phase A, which should provide the RF plant for the new booster, and the choice of having the amplifier built by industry. Different choices have been compared as use of one single scientific klystron, the use of TV klystrons and IOTs. Following this analysis the IOT choice has been taken and the $150 \mathrm{~kW}$ amplifier is made combining two $80 \mathrm{~kW}$ IOTs transmitters.

Each transmitter is designed completely independent and can work standalone. This helps to increase operational flexibility since the fault or maintenance of one transmitter does not imply the complete stop of the plant, given that the other transmitter can continue to operate. A crowbarless technology for the high voltage power supply has been chosen.

The two transmitters will be combined by means of a switchless combiner. This device, made of two hybrids and a waveguide phase shifter, has the advantage of having only $3 \mathrm{~dB}$ loss in case one transmitter is out of service, instead of $6 \mathrm{~dB}$ as in a standard hybrid combiner. Due to the isolation, maintenance of one transmitter could be done while the other is in service. The main disadvantage is the increased size of the combining system. However in this case only two tubes have to be combined, therefore this is not a big issue.

Due to the power level and frequency involved the transmission of the RF power is accomplished by means of a WR1800 waveguide system. A waveguide to coaxial transition is mounted to interface with the coaxial cavity input power coupler.

\section{TECHNICAL DESCRIPTION}

\section{Power Amplifier}

The power amplifier has been ordered as a turnkey system to ELECTROSYS (Orvieto, Italy) according to 
Elettra's specifications. It is composed of two identical 80 $\mathrm{kW}$ transmitters, the waveguide switchless combiner and a common rack which routes the driving signals to the two transmitters and performs a global supervision of the amplifier [3]. Each transmitter can work standalone. By a proper setting of the combiner one can feed the cavity with the sum of the outputs of the two transmitters, the power delivered by one of them or send both the outputs to the combiner load.

The specified output power is $150 \mathrm{~kW}$ at $500 \mathrm{MHz}$ for $13 \mathrm{dBm}$ input power. The output power dynamics is higher than $35 \mathrm{~dB}$. The efficiency of the tubes at maximum cw output power is higher than $65 \%$

The RF chain of each transmitter is composed of a 400 W solid state LDMOS amplifier followed by a $80 \mathrm{~kW}$ IOT from Thales Electron Devices (TH793). The IOT is mounted on a trolley inside the transmitter rack. The input signal is applied between grid and cathode through a coaxial input cavity. The output power is extracted through a double tuned output cavity system.

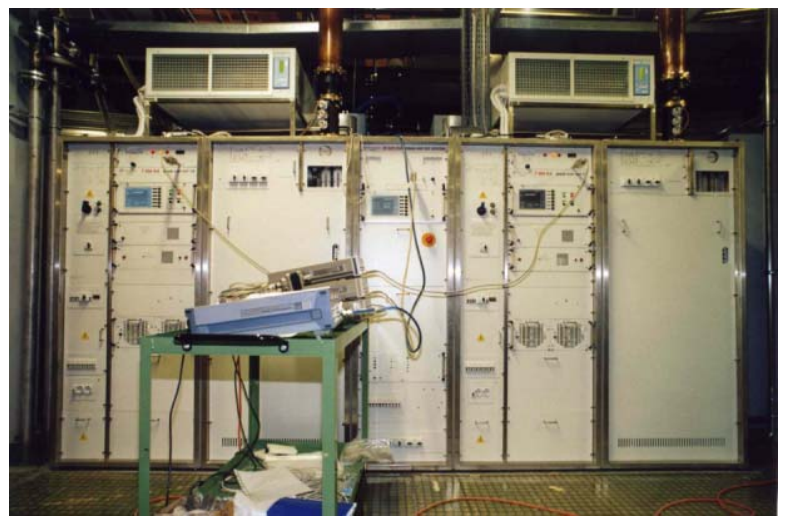

Figure 1: The RF power amplifier.

The high voltage power supply $(37 \mathrm{kV}, 3.8 \mathrm{~A})$ of each IOT is a switched mode power supply. This solution is more compact than standard HV power supplies and does not require the use of a crowbar tube. In addition the use of oil capacitors or transformers is avoided. On the technical side, the beam voltage is stabilised independently of output power variations and mains input variation, the first aspect being particularly important considering that the IOT is not a class A amplifier and that the required power depends on the current stored in the storage ring and its energy. Switching frequency of the power supply is $17 \mathrm{kHz}$ and the associated ripple is very low. However, the switching frequency can be adjusted in the range 16 to $21 \mathrm{kHz}$ [4], if it arises any problem of interaction with the stored beam spectrum.

The control system of each transmitter performs all the controls and actions needed for operation, monitor, fault handling and interfaces itself with the global supervision of the amplifier.

\section{Power Transmission}

The power amplifier is protected by a $200 \mathrm{~kW} 500$ $\mathrm{MHz}$ circulator provided by AFT (Germany). The circulator can sustain the whole forward and reflected power at the same time at any phase. The circulator load is a ferrite load provided by S.P.A. Ferrite (Russia). All remaining waveguide components are made by MEGA (USA). For interlock purposes, arc detectors are mounted on the circulator and on the $\mathrm{H}$ waveguide bends.

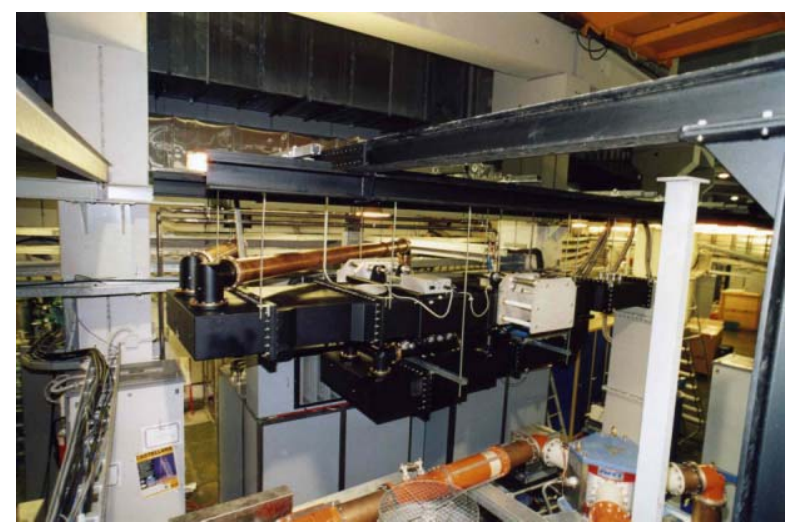

Figure 2: The switchless combiner.

\section{Waveguide to Coaxial Transition}

Although for the first months a standard waveguide to coaxial transition is used, in parallel a dedicated transition has been designed.

Water cooling for the inner conductor has been added to cope with the higher RF power, decreasing thus the probability of thermal breakdown in case of full reflection of the forward cavity power (as it could happen in case of fast beam losses and/or cavity mismatch). To access the inner conductor with cooling pipes, a WR1800 doorknob adapter has been developed. The expected return loss is below $40 \mathrm{~dB}$ at the working frequency. In addition to the requisite of performing an efficient cooling, the coaxial part of the transition has been designed in order to minimize the impact of the power from cavity higher order modes that could be excited by the beam.

The construction of the complete transition (by MEGA) is completed and the transition will be tested at full RF power during next summer at the RF lab of DESY (Germany).

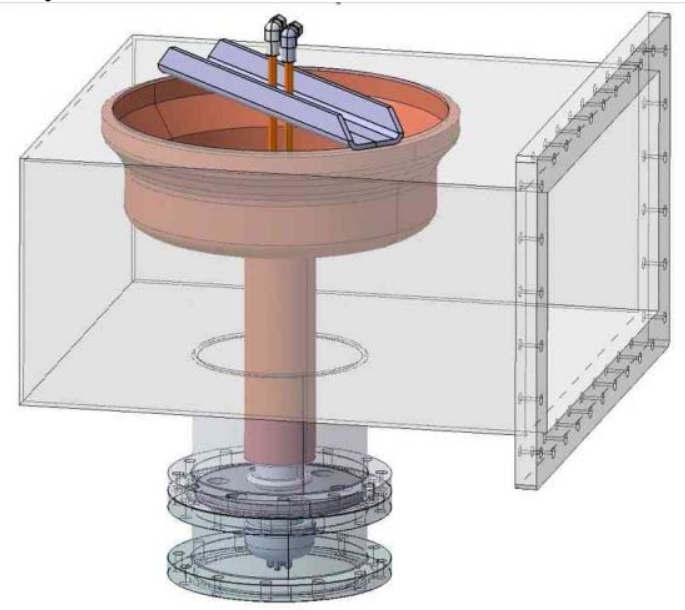

Figure 3: The waveguide to coaxial transition.

T06 Room Temperature RF 


\section{INSTALLATION}

All installations could be performed only during the planned machine shutdowns to avoid interference with the users' operation calendar, so they had to be well scheduled. During the first half of 2006 the upgrade of the electrical and cooling plants was performed and during summer the amplifier and all the components of the power plant were installed and tested.

The main results of the measurements performed on the amplifier are listed in table 1. All the required specifications were met. Figure 4 shows the transfer curve of the complete amplifier and of the two transmitters. It can be seen that the differences between the two transmitters are negligible. The ripple coming from the $\mathrm{HV}$ power supply (at 2 times the switching frequency) is below $69 \mathrm{~dB}$. Signal to noise spectrum is below $71 \mathrm{~dB}$. Second harmonic has been measured to be below $40 \mathrm{~dB}$ and third below $50 \mathrm{~dB}$.

Table 1: Amplifier Measurements

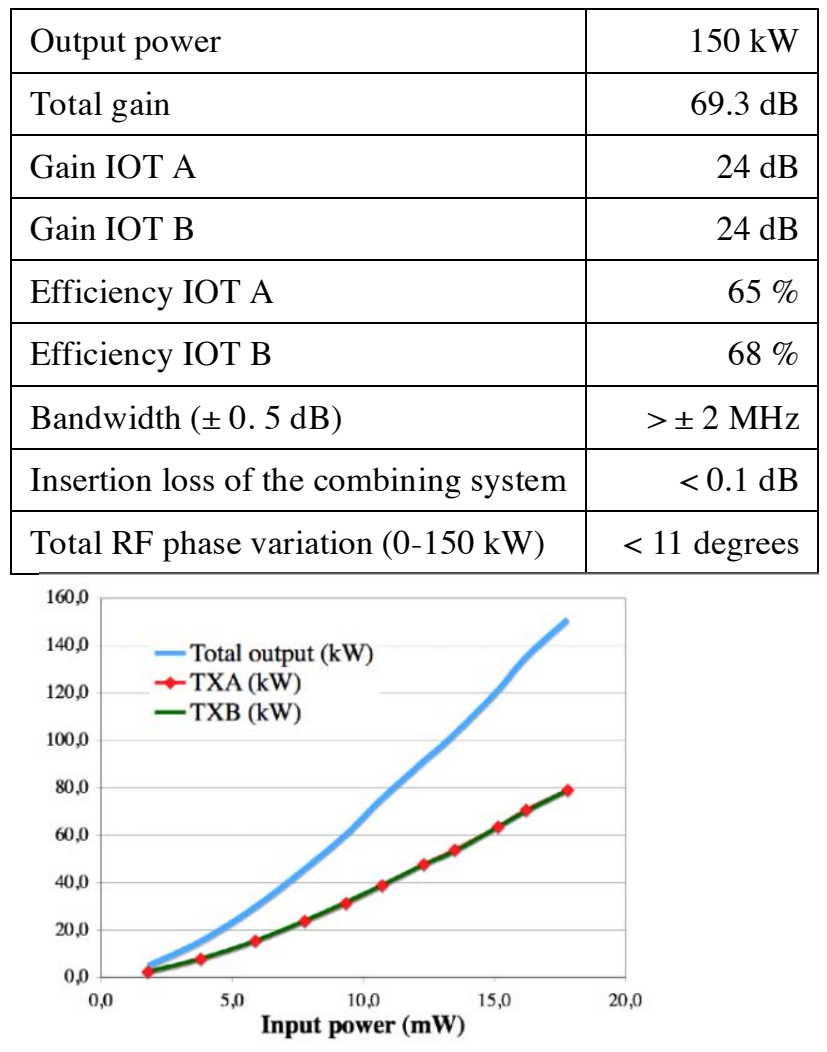

Figure 4: Transfer curves.

All the components of the power plant were tested at low and high power. The circulator was tested with the second port connected to a variable length high power short circuit, in order to test it at all phases between direct and reflected power.

\section{COMMISSIONING}

To assess the performances of the system and to debug it prior to the operation on the machine, a long-term test on dummy loads was planned during autumn 2006. The amplifier and the other components of the power plant were continuously working in parallel with machine operation 24 hours/day for 3.5 months. Power levels were set from 100 to $150 \mathrm{~kW}$. Following the tests, the main improvements that were done regarded the input cavity of the IOTs to improve the behaviour at high beam currents. The other major modification regarded the output coaxial line just after the tube, in order to avoid discharges in the line due to mismatch caused by the heat load at high power. These improvements were done by the tube manufacturer and the amplifier manufacturer respectively.

At the end of the dummy load test, the plant was connected to the cavity. Therefore from the first run of 2007 the cavity is powered by the new plant, as foreseen in the project schedule. Since the cavity has not yet been conditioned to input power levels higher than $60 \mathrm{~kW}$, the power delivered by the plant has been limited to this level during the first half of this year. For radiation safety reasons, the conditioning of the cavity can be done only during the shutdowns and with the access to the ring tunnel forbidden. Therefore it is almost impossible to perform it during the short shutdowns planned during the first 9 months of 2007 due to interference with other activities. At latest it will be done during the long shutdown foreseen at the end of 2007 for the connection of the new injector. At this point it will be possible to fully exploit the increased available power.

Up to now there has been no downtime due to the new plant, which is a nice indication of amplifier's reliability.

\section{CONCLUSIONS}

The first phase of the Elettra RF system upgrade project is almost concluded. Once the conditioning of the cavity will be completed, there will be a $25 \%$ increase in the total available RF power for cavities and beam. The cavity voltage in one cavity could be increased by a factor 1.4. This will also give a slight increase in beam lifetime, since in Elettra lifetime is Touschek dominated

Once the performance of the first phase will be assessed, the following phases will be evaluated also in relation with the other upgrades of the facility.

\section{ACKNOWLEDGEMENTS}

The contribution of G. Ocera from Electrosys and the collaboration of the staffs of Electrosys and Thales Electron Devices are gratefully acknowledged.

\section{REFERENCES}

[1] A. Fabris et al., "Elettra RF System Upgrade Project", EPAC'02, Paris, June 2002, p. 2142.

[2] A. Fabris et al., "RF System for the Elettra New Full Energy Booster Injector", this Conference.

[3] Electrosys , "B652 KA 150KW UHF IOT (1+1) Active reserve -Technical Manual", June 2006.

[4] G. Ocera, private communication. 